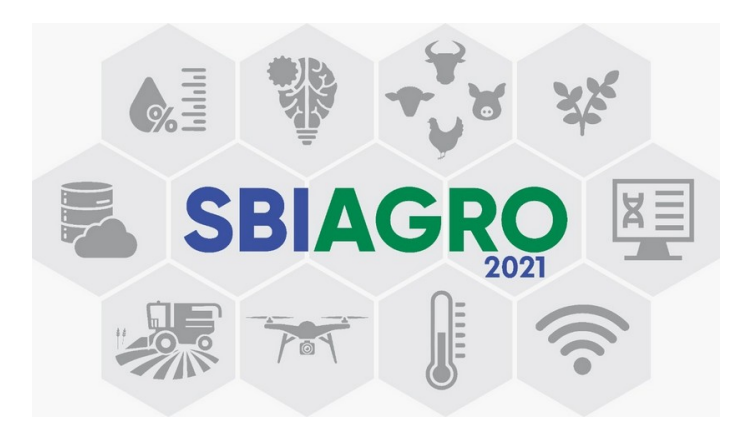

\title{
Semantic Sensor Network Ontology como modelo de referência em soluções de monitoramento agrícola
}

\author{
Julio C. Nardi ${ }^{1}$, Vitor N. Alves ${ }^{1}$, Carlos A. R. Junior ${ }^{1}$, Fabiano B. Ruy ${ }^{2}$, Robson \\ Prucoli Posse ${ }^{3}$ \\ ${ }^{1}$ Instituto Federal do Espírito Santo (Ifes) - Campus Colatina \\ Av. Arino Gomes Leal, no 1700, Santa Margarida, Colatina-ES, Brasil \\ ${ }^{2}$ Instituto Federal do Espírito Santo (Ifes) - Campus Serra \\ Rodovia ES-010, Km 6,5 - Manguinhos, Serra-ES, Brasil \\ ${ }^{3}$ Instituto Federal do Espírito Santo (Ifes) - Campus Itapina \\ Rodovia BR-259, Km 70, Colatina-ES, Brasil \\ julionardi@ifes.edu.br, vitornicchioalves@gmail.com,
junior_recla@hotmail.com, ffabianoruy, robson.posse\}@ifes.edu.br
}

\begin{abstract}
Solutions of Internet-of-Things in Agriculture deal with devices and software applications that do not share the same information model. This limits the semantic interoperability between them. This work presents and discusses the use of SSN ontology (W3C) in two initiatives related to monitoring of environmental variables. Thus, it aims at supporting data integration of devices, and development of software applications more interoperable, in so far they use a conceptual reference model.
\end{abstract}

Resumo. Soluções de Internet das Coisas na Agricultura lidam com dispositivos e aplicações de software que não compartilham um mesmo modelo de informação. Isso limita a interoperabilidade semântica entre eles. Este trabalho apresenta e discute o uso da ontologia SSN (W3C) em duas iniciativas relacionadas ao monitoramento de variáveis ambientais. Como resultado, busca-se promover integração de dados de dispositivos e desenvolvimento de aplicações de software mais interoperáveis, na medida em que se utiliza um modelo conceitual de referência.

\section{Introdução}

As áreas de Agricultura de Precisão (AP) e Internet das Coisas (Internet of Things IoT) têm se aproximado cada vez mais na medida em que o monitoramento automático de eventos e variáveis ambientais tem levado ao aumento da produção agrícola e da redução do consumo de recursos. Por meio de dispositivos interconectados e associados a aplicações de softwares é possível capturar, manipular e compartilhar dados reais de condições de solo, clima e outros para otimizar a produção [Shafi et al., 2019].

Esse contexto se caracteriza pelo uso de diversos dispositivos e aplicações "heterogêneos", desenvolvidos por diferentes fabricantes e sem compartilharem um 
mesmo modelo de informação. Como consequência, um dos grandes desafios, reconhecidos tanto pela Indústria [Ericsson Blog, 2021] quanto pela Academia [Barnaghi et al., 2012] [IERC, 2015] [Honti and Abonyi, 2019], é a promoção de "interoperabilidade semântica" entre dispositivos, entre aplicações e entre dispositivos e aplicações em soluções de IoT. Para além da integração em nível técnico (de hardware e de plataforma), por meio da promoção da interoperabilidade semântica busca-se alinhar o significado dos dados trocados entre dispositivos e aplicações para que haja o correto entendimento desses dados, a devida comunicação e o uso efetivo.

Modelos conceituais de referência e padrões têm sido desenvolvidos e adotados [Honti and Abonyi, 2019], tais como: IoT-A Reference Model [Bauer et al., 2013], The Internet of Things Reference Model [CISCO, 2014] e IoT-Lite Ontology [W3C, 2005]. Tais modelos/padrões buscam estabelecer um referencial conceitual e uma interlíngua a ser adotada na integração de dispositivos e aplicações de software. A ontologia SSN (Semantic Sensor Network) [W3C, 2017] é um padrão internacional amplamente utilizado cujo objetivo é descrever o domínio de sensoriamento abordando conceitos como sensores, suas observações, os procedimentos envolvidos, as propriedades observadas, os atuadores e suas ações etc. Ontologias têm sido reconhecidas como um importante artefato para enriquecer os dados sensoriados e promover interoperabilidade semântica [Honti and Abonyi, 2019]. Assim, SSN tem sido aplicada em diferentes cenários aqueles relacionados à Agricultura de Precisão [W3C, 2017] [Li et al., 2018].

Entretanto, o uso de ontologias para promoção de interoperabilidade semântica, seja na construções de soluções de software ou na integração de dados requer sistematicidade. Abordagens que tratam da derivação/transformação de ontologias em esquemas de banco de dados [Guidoni et al., 2020] e da integração de dados de aplicações e/ou dispositivos têm sido desenvolvidas [Honti and Abonyi, 2019], mas ainda se tem que avançar no entendimento e na adoção de abordagens dessa natureza.

Este trabalho, portanto, apresenta e discute o uso de SSN em duas iniciativas relacionadas ao monitoramento de variáveis ambientais: (i) desenvolvimento de aplicação de software para monitoramento online e (ii) realização de integração de dados sensoriados advindos de arquivos (.XLS). São discutidos aspectos relacionados: (i) a derivação de modelo de classes a partir da ontologia SSN e (ii) a alinhamento semântico entre estruturas de dados geradas por dispositivos heterogêneos e um modelo de referência (SSN) visando a integração de dados. A partir dos resultados deste trabalho, espera-se contribuir para o desenvolvimento de outras iniciativas correlatas, em especial, aquelas baseadas em ontologias, uma vez que pesquisas atuais evidenciam o uso de ontologias como um caminho promissor no sentido de obter soluções de IoT mais adaptáveis e interoperáveis [Honti and Abonyi, 2019].

\section{Referencial Teórico}

Interoperabilidade semântica no âmbito de soluções de IoT tem sido foco de vários trabalhos [Honti and Abonyi, 2019] [Drury et al., 2019]. Tais soluções, em geral, fazem uso de inúmeros sensores, formando uma rede de dispositivos que apresentam capacidades complementares e especificações distintas, requerendo abordagens de integração no nível semântico para alinhar a troca de dados entre eles [Li et al., 2018]

Nesse contexto, a interoperabilidade semântica se coloca como um dos grandes desafios em soluções IoT, na medida em que o enriquecimento semântico de dados sensoriados brutos (raw sensory data) é crucial para suportar integração de informação, que levará à produção de conhecimento e à inovação [Honti and Abonyi, 2019].

Também no domínio de Agricultura de Precisão, abordagens e tecnologias semânticas de várias naturezas tem sido desenvolvidas [Honti and Abonyi, 2019] [Drury et al., 2019], dentre as quais estão aquelas relacionadas à integração e compartilhamento de dados sensoriados. Nesse contexto, a (re)utilização de ontologias e 
modelos de referência como "recursos semânticos" se destaca [Drury et al., 2019]. Mais especificamente, ontologias se apresentam como modelos conceituais que buscam, dentre outros, minimizar problemas de comunicação (p.ex., ambiguidade) entre dispositivos, sistemas, pessoas e organizações [Guizzardi, 2007].

A ontologia SSN (Semantic Sensor Network), como um padrão internacional, é amplamente utilizada. Como tal, busca formalizar, explicitamente, conceitos e relações de um determinado domínio (sensoriamento), que são compartilhados ("consensuais") dentro de uma comunidade [Gruber, 1993].

A Figura 1 apresenta um fragmento de SSN cujo foco é na descrição de conceitos relacionados à observação de uma determinada propriedade. $\mathrm{O}$ fragmento descreve, em linhas gerais, que sensores (Sensor) fazem observações (Observation), que estão relacionadas a propriedades observáveis (ObservableProperty) e utilizam determinados procedimentos (Procedure). Além da representação gráfica (ilustrada pela Figura 1), SSN conta com uma formalização em OWL (Ontology Web Language), permitindo também sua utilização/processamento por máquinas.

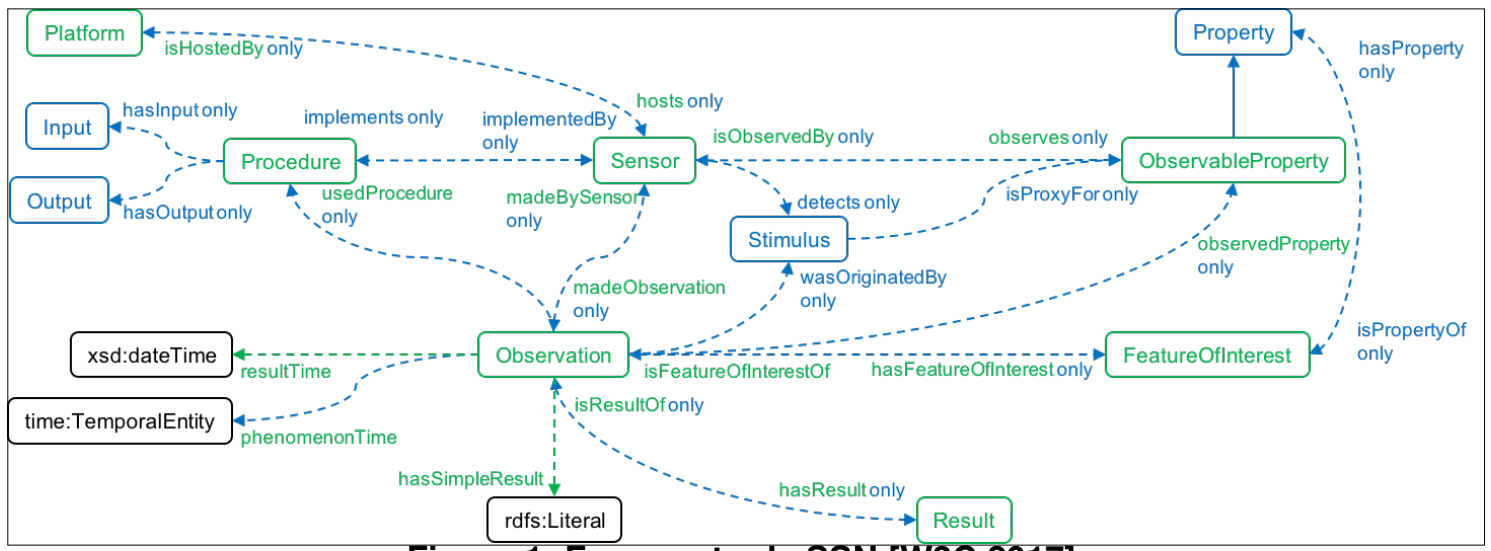

Figura 1: Fragmento de SSN [W3C 2017].

Ontologias, como no caso de SSN, têm sido utilizadas para suportar o desenvolvimento e à integração de sistemas de softwares e soluções IoT, que necessitam lidar com um determinado domínio [Abinaya et al., 2015] [Nardi et al., 2013] [Honti and Abonyi, 2019]. Dessa forma, o conhecimento compartilhado e explicitado em uma ontologia é reutilizado na medida em que é incorporado nos sistemas de software, p.ex., por meio de transformação de modelos [Guidoni et al., 2020] dando origem a modelos de classes, modelos relacionais, regras de negócio etc. Como resultado, espera-se que tais soluções tenham um maior grau de reuso e interoperabilidade, por fazerem uso de dados/informações alinhados a modelos conceituais "consensuais".

\section{Da Ontologia SSN para um Modelo de Informação}

Com vistas à utilização do conhecimento formalizado em SSN como referência na construção de soluções de software, o primeiro passo foi a derivação de um modelo de classes usando a UML (Unified Modeling Language), conforme ilustra a Figura 2.

O processo de derivação é iterativo, incorporando, gradualmente, os conceitos, relações e restrições da ontologia no modelo de classes. Pode-se entender esse processo como uma espécie de "transformação de modelos" [Guidoni et al., 2020], na medida em que o modelo ontológico de SSN é transformado em um modelo de classes UML. Ao se realizar a transformação, construtos da linguagem de representação do modelo de origem são mapeados em construtos da linguagem de representação do modelo de destino. Assim, conceitos, relações e restrições (da ontologia) são representados no modelo resultante (de classes) acomodando-se de acordo com a expressividade da linguagem utilizada. Ademais, como derivou-se um modelo de classes visando a 
construção de soluções de software, alguns aspectos de design de software foram inseridos (p.ex., tipos e navegabilidades). A Figura 3 apresenta um fragmento do modelo de classes gerado.

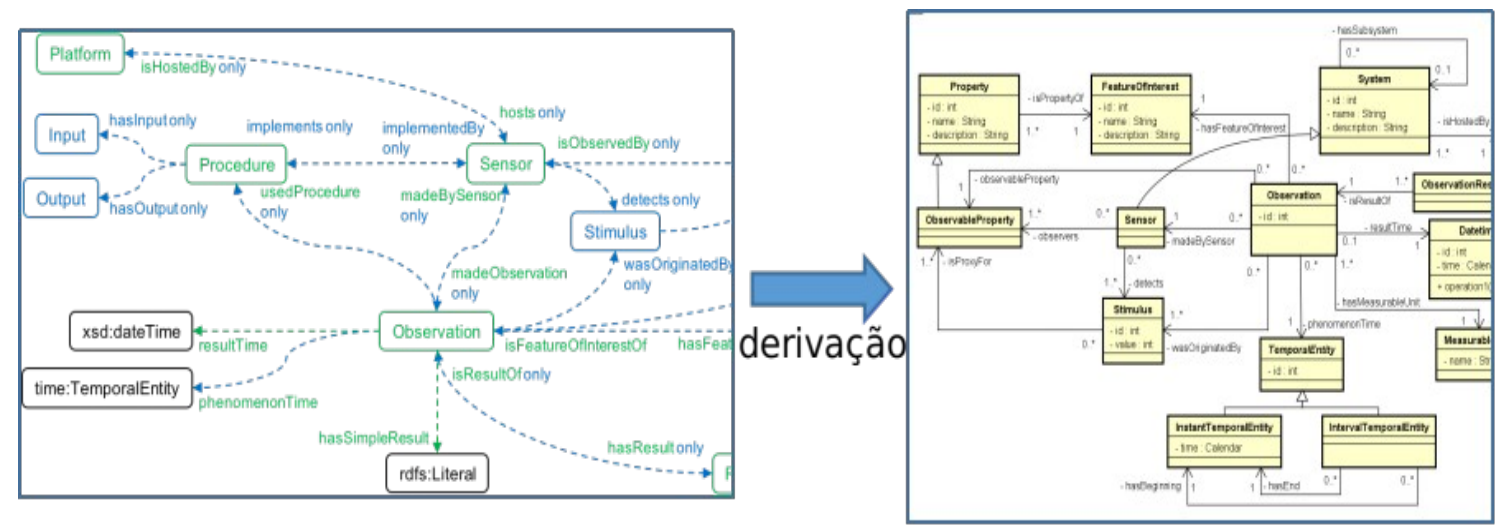

Figura 2: llustração da derivação de SSN em um modelo de classes.

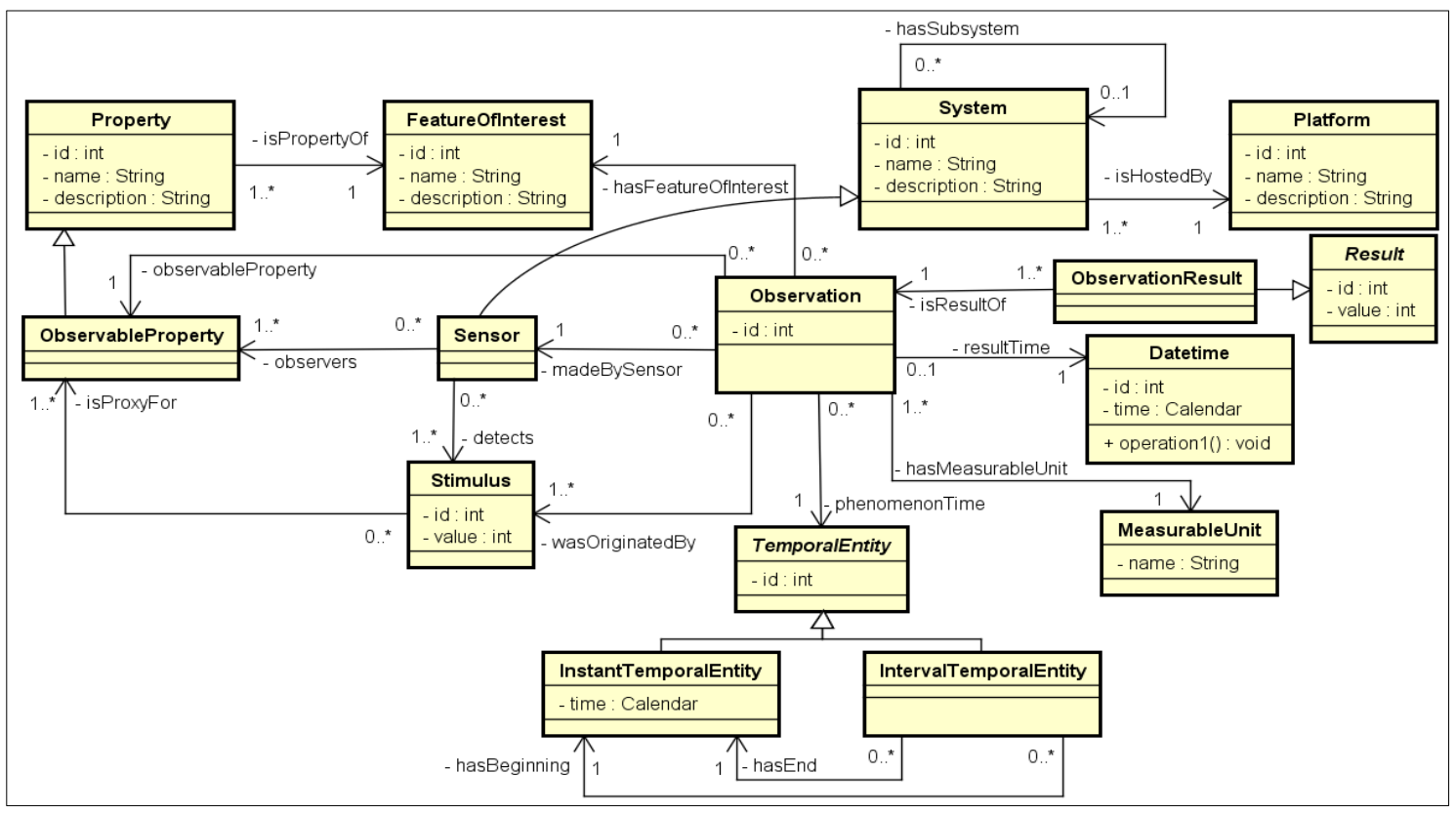

Figura 3: Fragmento do modelo de classes gerado a partir de SSN.

De acordo com o modelo, uma Platform (p.ex., um viveiro de mudas) hospeda/contem vários System. System é um conceito especializado em Sensor. Um Sensor (um sensor de umidade) observa uma ou várias ObservableProperty (umidade). Uma Observation (leitura da umidade do solo em um dado momento t1) é feita por um Sensor, sendo originada por um Stimulus (acionamento de um temporizador), que é detectado pelo Sensor. Uma Observation pode acontecer instantaneamente ou ao longo de um intervalo temporal, gera ObservationResults (20, valor sensoriado de umidade). ObservableProperty é uma especialização do conceito Property, que, por sua vez, é uma propriedade de uma FeatureOfInterest (solo do viveiro de mudas).

\subsection{Iniciativa 1: SSN Aplicada no Desenvolvimento de Aplicação de Software}

Nesta iniciativa foi construída uma solução de software para monitoramento de irrigação usando sensores capturam dados de variáveis de ambiente como temperatura do ar e umidade do solo e os transmitem para um middleware que, por meio de serviços de software, os envia a uma aplicação web que realiza o armazenamento. De posse 
desses dados, o usuário pode realizar o monitoramento remoto das variáveis ambientais usando aplicações específicas em diferentes plataformas (p.ex., computador e celular).

Conforme apresenta a Figura 4, o sistema foi estruturado em 4 (quatro) camadas: a Camada de Sensoriamento (onde os sensores se encontram localizados no ambiente monitorado), a Camada de Conexão (possui um middleware rodando em um Raspberry Pi, que estabelece a conexão entre os sensores e a aplicação de software), a Camada de Gerenciamento (onde se encontra a aplicação de software (JavaEE) juntamente com o banco de dados (MySQL)) e a Camada de Aplicação do Usuário (onde o usuário realiza o monitoramento por meio de aplicações de software - desktop e mobile). Todo o setup da solução como, por exemplo, cadastros dos sensores, plataforma, medidas e propriedades observadas são realizados por um usuário administrador. Em tempo real, os dados sensoriados são lidos e enviados, via serviços web, à aplicação, a qual os analisa, identifica a origem, o que representam e, assim, os armazena na base de dados.

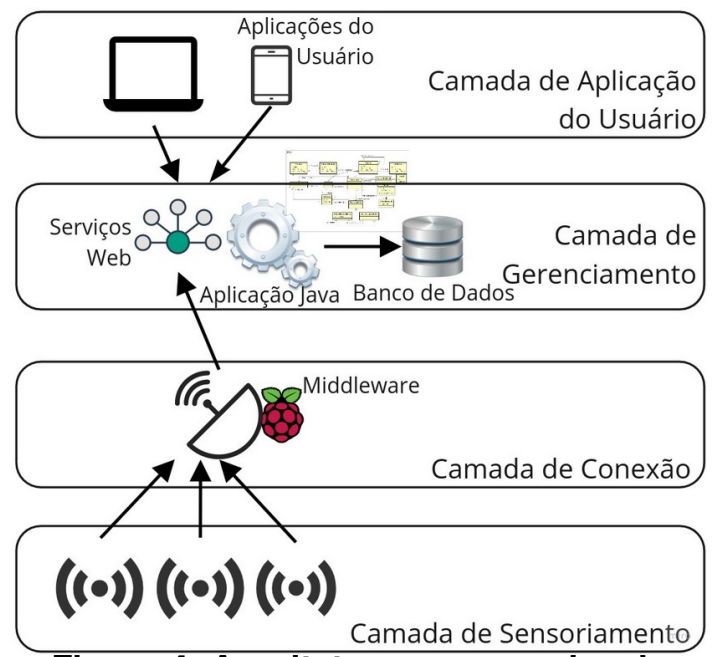

Figura 4: Arquitetura em camadas da solução proposta.

Para implementação das classes de domínio, dos serviços web (REST) e da estrutura da base de dados, utilizou-se o modelo de classes derivado de SSN (vide Figura 3), fornecendo, dessa forma, a referência conceitual provida pela ontologia. Assim, o banco de dados armazena tanto os dados sensoriados quanto as configurações de setup da infraestrutura de sensoriamento como, por exemplo, marca/modelo dos dispositivos utilizados. A Figura 5 ilustra a implementação da classe Sensor na aplicação de software baseada em SSN.

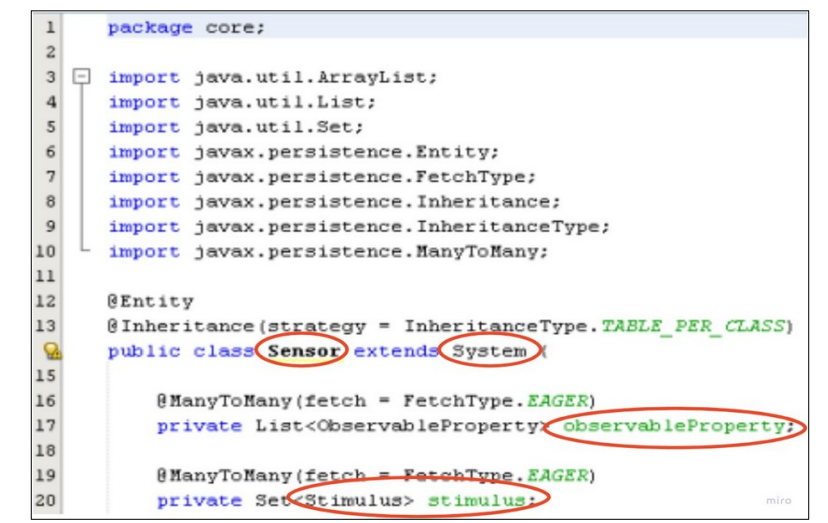

Figura 5: Exemplo de implementação baseada em SSN. Classe Sensor.

A classe Sensor, que herda de System, tem relação com duas outras classes 
implementadas: ObservableProperty e Stimulus. Assim, pode-se observar que a implementação em código fonte (Java) mapeia os mesmos conceitos e relações do modelo de classe, que, por sua vez, foram derivados de SSN.

\subsection{Iniciativa 2: SSN Aplicada na Integração de Dados de Diferentes Dispositivos}

Nesta iniciativa, usou-se o modelo de classes gerado a partir de SSN para criar uma aplicação que realizasse a leitura de dados fornecidos em arquivos .XLS gerados a partir de dois dispositivos utilizados no monitoramento de condições climáticas/ambientais - (i) uma estação climática HOBO® U30 Station; e (ii) um dispositivo Decagon ${ }^{\circledR}$ EM43375 - e inserisse esses dados em um banco de dados único.

O processo de construção da solução consistiu, inicialmente, no entendimento das estruturas e dados dos arquivos gerados por cada dispositivo. Em seguida, passou-se para a construção do alinhamento semântico entre os dados e estrutura de cada arquivo e o modelo de classes gerado à luz de SSN. Isso se faz necessário na medida em que os dados dos arquivos são importados para uma base de dados única, cuja estrutura é baseada no modelo de classes de SSN. Deve haver uma correspondência entre cada dado contido nos arquivos e as estruturas de informação (classes e tabelas) correspondentes da solução. O modelo de classes baseado em SSN foi utilizado como modelo de referência para o alinhamento semântico. As figuras 6 e 7 ilustram os mapeamentos entre os dados e estruturas de cada arquivo e o modelo de classes.

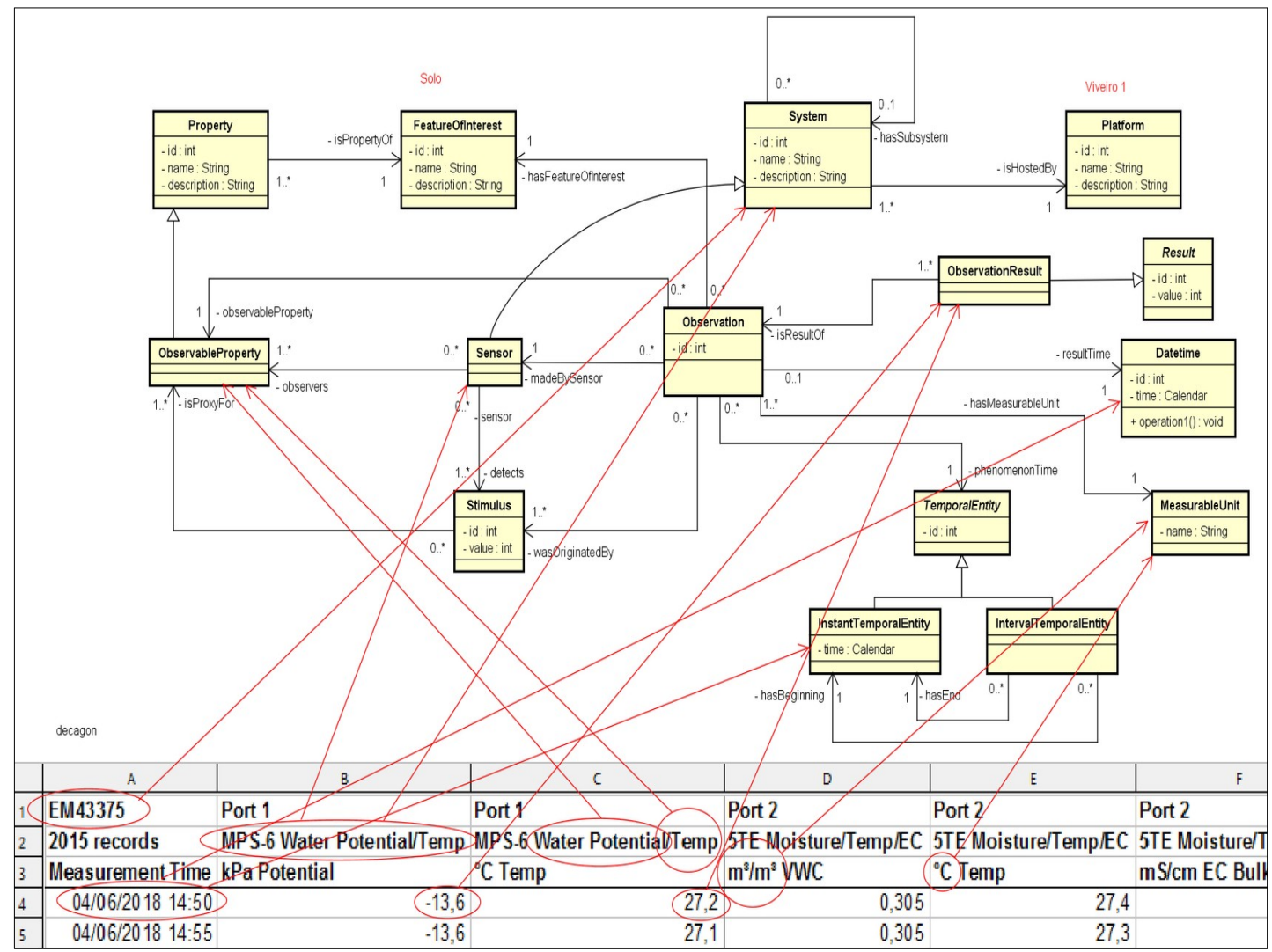

Figura 6: Mapeamentos conceituais entre a planilha de dados do Decagon $\AA$ e 0 modelo de classes.

Após a definição dos mapeamentos, a aplicação de software foi implementada. As classes de domínio (em Java) e a estrutura da base de dados relacional (MySQL) refletiram o modelo de classes baseado em SSN. Em seguida, foi implementado o módulo de importação, que tem a função ler cada arquivo de dados e, com base nos mapeamentos, realizar a persistência. Assim, ao final da importação, todos os dados 
foram inseridos na base de dados, considerando, agora, o mesmo modelo de informação fornecido pela ontologia. Isso, tende a facilitar consultas e análises dos dados, então, integrados. A Figura 8 ilustra a arquitetura de alto nível da solução.

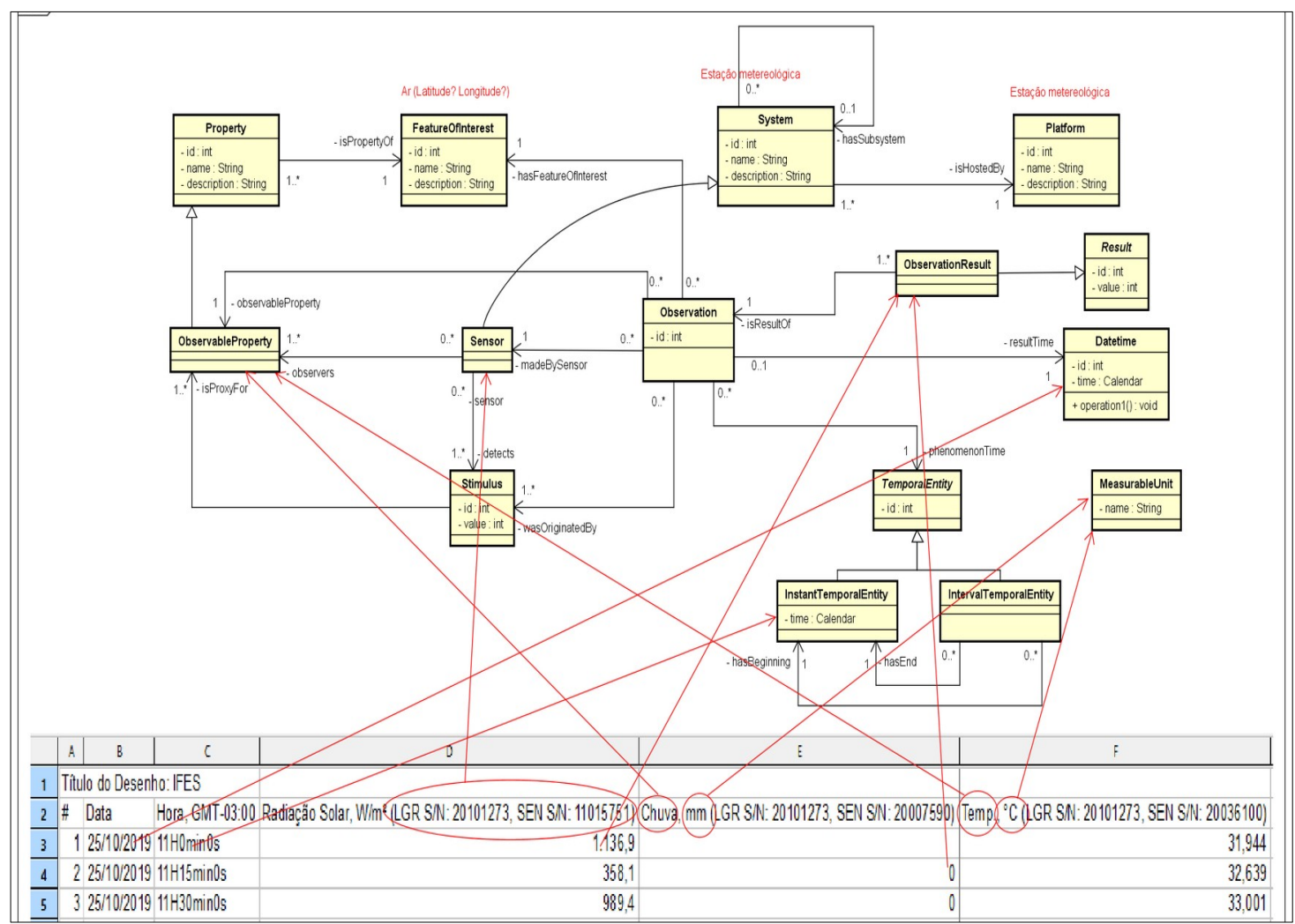

Figura 7: Mapeamentos conceituais entre a planilha de dados da estação e o modelo de classes.

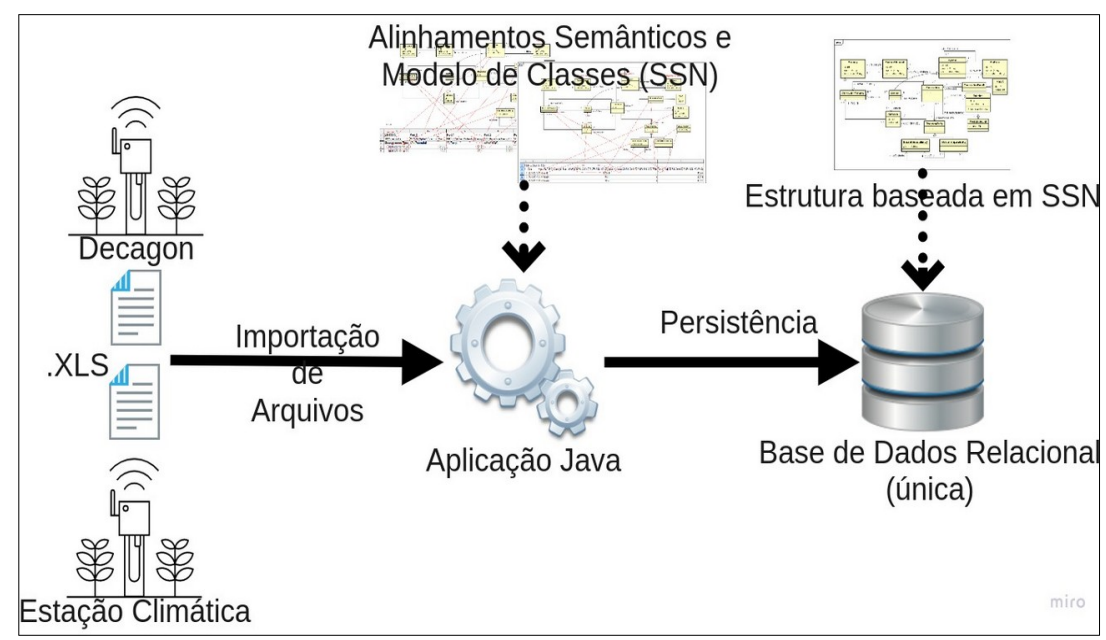

Figura 8: Arquitetura de alto nível da solução.

\section{Discussão}

Considerando o processo de derivação do modelo de classes a partir de SSN e o desenvolvimento das 2 (duas) iniciativas, alguns aspectos merecem destaque.

Aspecto 1. A ontologia SSN apresenta, basicamente, 3 (três) perspectivas: Observation, 
Actuation e Sampling. As iniciativas apresentadas neste trabalho focaram na perspectiva Observation, devido ao seus requisitos. De todo modo, uma vez que SSN estabelece uma estrutura padrão entre suas perspectivas, acredita-se que avançar na ampliação do modelo de classes derivado rumo às duas outras perpectivas (Actuation e Sampling) seja análogo ao processo apresentado neste trabalho.

Aspecto 2. Ao derivar o modelo de classes, foram incluídos atributos mínimos (p.ex., IDs, nome e descrição), para dar suporte ao aspecto informacional do modelo. Ademais, foram definidas navegabilidades, para atender a requisitos não funcionais como, por exemplo, economia de memória. De todo modo, tais alterações não descaracterizam SSN. No caso de atributos, entende-se que, havendo necessidade, pode-se especializar o modelo incluindo novas classes que venham a acrescentar eventuais atributos/relações. Com a especialização, é possível manter a conceitualização fundamental baseada em SSN e, mesmo assim, adequar o modelo a cenários específicos.

Aspecto 3. Em relação ao conceito TemporalEntity, existente em SSN, esse foi especializado no modelo de classes gerado em dois outros conceitos: InstantTemporalEntity e IntervalTemporalEntity. Tal ajuste foi julgado necessário para melhor descrever os possíveis tipos de momentos em que um fenômeno pode ocorrer, ou seja: instantaneamente ou durante um intervalo de tempo. De todo modo, a superclasse TemporalEntity, mantém relação direta com conceito original de SSN.

Aspecto 4. A tarefa de mapeamento entre cada arquivo de dados gerado por um modelo/marca de dispositivo e o modelo de classes utilizado precisa ser realizada unicamente. Isso é feito pelo modelador/desenvolvedor com base na especificação técnica do dispositivo e com o apoio de um especialista de domínio (p.ex., um agrônomo que conhece os dados sensoriados). Essa tarefa, quando realizada para uma primeira solução, consome tempo. Depois, entretanto, o mapeamento poderá ser reutilizado em outras iniciativas que vierem a fazer uso do mesmo modelo/marca de dispositivo e mesmo modelo de classes. Daí a importância de se utilizar um modelo de referência. Pode-se pensar em uma abordagem semelhante ao uso drivers (dispositivo driver - plataforma). Cada dispositivo terá o seu driver de importação/mapeamento dos dados sensoriados para uma base de dados padrão/referência, otimizando seu uso.

\section{Conclusões}

Este trabalho discute o uso de SSN em duas iniciativas relacionadas ao monitoramento de variáveis ambientais na Agricultura: (i) desenvolvimento de aplicação de software para monitoramento online e (ii) integração de dados sensoriados advindos de arquivos (.XLS) em uma base de dados única. A despeito das particularidades de cada iniciativa, há uma abordagem comum cujas etapas merecem destaque: (i) recepção do dado sensoriado; (ii) caracterização do dado à luz de um modelo conceitual de referência (SSN); e (iii) uso e armazenamento do dado em uma base de dados compartilhada.

Dentre as contribuições do trabalho destacam-se: (i) derivação da ontologia SSN em um modelo de classes aplicado nas iniciativas apresentadas e que pode ser reutilizado em outras iniciativas correlatas; (ii) estratégia para mapeamento de dados de diferentes fontes de sensoriamento para um modelo comum (de referência); e (iii) discussão e criação de bases de dados alinhadas a uma ontologia padrão W3C (SSN), o permite que consultas e análises de dados sejam feitas à luz de conceitos mais amplamente aceitos pela Academia e Indústria.

Como trabalhos futuros espera-se: (i) integrar as soluções das duas iniciativas apresentadas em uma plataforma única de monitoramento, (ii) aplicar tal plataforma no âmbito de culturas agrícolas e na produção de mudas, (iii) ampliar o modelo de classes gerado a fim de comtemplar também as perspectivas Actuation e Sampling de SSN e (iv) avançar nas pesquisas e aplicações de SSN, buscando propor melhorias na especificação padrão da ontologia. 


\section{Referências}

Abinaya, Kumar, V., \& Swathika. (2015). "Ontology Based Public Healthcare System in Internet of Things (IoT)". Procedia Computer Science, 50, 99-102. https://doi.org/http://dx.doi.org/10.1016/j.procs.2015.04.067

Barnaghi, P., Wang, W. E. I., Henson, C., \& Taylor, K. (2012). "Semantics for the Internet of Things: early progress and back to the future". International Journal on Semantic Web and Information Systems. https://doi.org/10.4018/jswis.2012010101.

Bauer, M., Bui, N., De Loof, J., Magerkurth, C., Nettstra“ter, A., Stefa, J., \& Walewski, J. W. (2013). "IoT Reference Model". In A. Bassi, M. Bauer, M. Fiedler, T. Kramp, R. van Kranenburg, S. Lange, \& S. Meissner (Eds.), Enabling Thing to Talk: Designing IoT solutions with the IoT Architectural Reference Model (p. 352). Springer.

CISCO. (2014). The Internet of Thing Reference Modelo (2014).

Drury, B., Fernandes, R., Moura, M.-F., \& Lopes, A. de A. (2019). "A survey of semantic web technology for agriculture". Information Processing in Agriculture, $6,487-501$.

Ericsson Blog. (2021). What is semantic interoperability in IoT and why is it important? https://www.ericsson.com/en/blog/2020/7/semantic-interoperability-iniot

Gruber, T. R. (1993). "A Translation Approach to Portable Ontologies". Knowledge Acquisition, 5(2), 199-220.

Guidoni, G. L., Almeida, J. P. A., \& Guizzardi, G. (2020). "Transformation of Ontology-Based Conceptual Models into Relational Schemas". Lecture Notes in Computer Science (Including Subseries Lecture Notes in Artificial Intelligence and Lecture Notes in Bioinformatics), 315-330. https://doi.org/10.1007/978-3-03062522-1_23

Guizzardi, G. (2007). “On Ontology, ontologies, Conceptualizations, Modeling Languages, and (Meta)Models”. In O. Vasilecas, J. Edler, \& A. Caplinskas (Eds.), Frontiers in Artificial Intelligence and Applications, Databases and Information Systems IV (Vol. 15, pp. 18-39). IOS Press.

Honti, G. M., \& Abonyi, J. (2019). "A Review of Semantic Sensor Technologies in Internet of Things Architectures". Hindawi. Complexity., 2019, 1-22. https://doi.org/https://doi.org/10.1155/2019/6473160

IERC. (2015). Internet of Things - IoT Semantic Interoperability: Research Challenges, Best Practices, Recommendations and Next Steps.

Li, S., Chen, L., \& Chen, S. (2018). "An SNN Ontology Based Environment Monitoring Method for Intelligent Irrigation System". Journal of Shanghai Jiaotong University (Science), 23(4), 577-583. https://doi.org/10.1007/s12204018-1959-0 
Nardi, J. C., Falbo, R. de A., \& Almeida, J. P. A. (2013). "Panorama of the Semantic EAI Initiatives and the Adoption of Ontologies by these Initiatives". International IFIP Working Conference on Enterprise Interoperability (IWEI 2013), 198-211.

Shafi, U., Mumtaz, R., García-Nieto, J., Hassan, S. A., Zaidi, S. A. R., \& Iqbal, N. (2019). "Precision Agriculture Techniques and Practices: From Considerations to Applications". Sensors (Basel), 19(17), 3796. https://doi.org/10.3390/s19173796

W3C. (2005). IoT-Lite Ontology. W3C Member Submission 26. https://www.w3.org/Submission/iot-lite/

W3C. (2017). Semantic Sensor Network Ontology (SSN). https://www.w3.org/TR/vocab-ssn/ 